\title{
Effect of one stretch a week applied to the immobilized soleus muscle on rat muscle fiber morphology
}

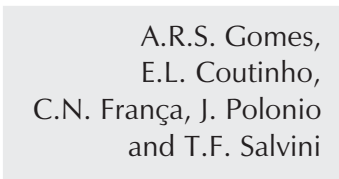

Unidade de Plasticidade Muscular, Departamento de Fisioterapia, Universidade Federal de São Carlos, São Carlos, SP, Brasil

\section{Correspondence \\ T.F. Salvini \\ Departamento de Fisioterapia \\ UFSCar \\ 13565-905 São Carlos, SP \\ Brasil \\ Fax: +55-16-3351-8345 \\ E-mail: tania@power.ufscar.br \\ Partial results of this study \\ were presented in abstract form \\ at the XVII Annual Meeting of the \\ Federação de Sociedades de Biologia \\ Experimental, Salvador, BA, Brazil, \\ August 28-31, 2002. \\ Research supported by FAPESP. \\ A.R.S. Gomes and J. Polonio are recipients of FAPESP fellowships. E.L. Coutinho and C.N. França are recipients of CAPES and CNPq fellowships.}

Received August 7, 2003

Accepted June 8, 2004

$\ldots \ldots \ldots \ldots \ldots \ldots \ldots$

\section{Abstract}

We determined the effect of stretching applied once a week to the soleus muscle immobilized in the shortened position on muscle fiber morphology. Twenty-six male Wistar rats weighing $269 \pm 26 \mathrm{~g}$ were divided into three groups. Group I, the left soleus was immobilized in the shortened position for 3 weeks; group II, the soleus was immobilized in the shortened position and stretched once a week for 3 weeks; group III, the soleus was submitted only to stretching once a week for 3 weeks. The medial part of the soleus muscle was frozen for histology and muscle fiber area evaluation and the lateral part was used for the determination of number and length of serial sarcomeres. Soleus muscle submitted only to immobilization showed a reduction in weight ( $44 \pm 6 \%, \mathrm{P}=0.002)$, in serial sarcomere number $(23 \pm 15 \%)$ and in cross-sectional area of the fibers $(37 \pm 31 \%, \mathrm{P}<0.001)$ compared to the contralateral muscles. The muscle that was immobilized and stretched showed less muscle fiber atrophy than the muscles only immobilized $(\mathrm{P}<0.05)$. Surprisingly, in the muscles submitted only to stretching, fiber area was decreased compared to the contralateral muscle ( $2548 \pm 659 v s 2961 \pm 806 \mu \mathrm{m}^{2}$, respectively, $\left.\mathrm{P}<0.05\right)$. In conclusion, stretching applied once a week for $40 \mathrm{~min}$ to the soleus muscle immobilized in the shortened position was not sufficient to prevent the reduction of muscle weight and of serial sarcomere number, but provided significant protection against muscle fiber atrophy. In contrast, stretching normal muscles once a week caused a reduction in muscle fiber area.

\section{Introduction}

An important question for rehabilitation is how to protect skeletal muscle from the effects of immobilization, especially muscles well known that skeletal muscle is an adaptable tissue which, when immobilized in the shortened position for 3 weeks, presents loss in the number of serial sarcomeres, muscle immobilized in the shortened position. It is
Key words - Muscle

- Stretching

- Immobilization

- Sarcomere number

- Muscle fiber area fiber atrophy and proliferation of connective tissue (1-3). These changes contribute to the significant reduction of the joint range of motion when the immobilization is removed.

The loss of serial sarcomeres in muscles immobilized in the shortened position occurs mainly after two weeks of immobilization (3). In contrast, muscles immobilized in the lengthened position for the same period of time show an increased number of serial 
sarcomeres, moderate or absent atrophy and no significant changes in connective tissue (2-7). It is also known that stretching of skeletal muscle is very important not only to increase the joint range of motion but also to prevent and treat injuries, representing a tool of therapeutic intervention for physical rehabilitation and sports medicine (for a review, see Ref. 7). Muscle stretching is recognized as a very powerful stimulant of muscle growth and protein synthesis (for reviews, see Refs. 8,9).

Several studies have demonstrated the importance of stretching to prevent connective tissue proliferation, muscle fiber atrophy, and the loss of serial sarcomeres in immobilized muscles $(6,8,9)$. A previous report showed that daily sessions of passive stretching applied for $30 \mathrm{~min}$ to the soleus muscle of rats immobilized in the shortened position were enough to prevent the loss of serial sarcomeres and to maintain the joint range of motion (2). However, $15 \mathrm{~min}$ of stretching applied every 2 days was not effective in preventing the loss of serial sarcomeres (6).

Recently, we evaluated the effect of 3 stretching sessions a week applied for 40 min to the soleus muscle of rats immobilized in the shortened position. Although this stretching frequency and duration did not prevent the loss of serial sarcomeres, it provided significant protection against muscle fiber atrophy, suggesting that muscle stretching regulates in a different manner the number of serial sarcomeres and the cross-sectional area of muscle fibers (Coutinho EL, Gomes ARS, França CN and Salvini TF, unpublished results). In the same study, when sessions of stretching were applied to nonimmobilized muscles there was an increase in both serial sarcomere number and muscle fiber area, reflecting an important hypertrophic effect.

Sessions of stretching are commonly used for humans during sports activities and rehabilitation programs to treat and prevent muscle shortening. Some reports on the procedures used in physical rehabilitation have described that one session of stretching per week is enough to maintain flexibility $(10,11)$. However, there are no detailed studies about the effect of one stretching a week on the morphology of the skeletal muscle fibers of shortened muscles and muscles of normal length.

Thus, the purpose of the present study was to determine the effects on muscle fiber morphology induced by one session of passive stretching a week applied to the soleus muscle of rats immobilized in the shortened position and also to non-immobilized muscles.

Although there are several differences between rodent and human skeletal muscles, mainly in terms of protein turnover, the results of the present study provide new information about the adaptation of skeletal muscle submitted to passive stretching sessions. To our knowledge, the present study is the first report of a protocol using this frequency and duration of muscle stretching applied to the skeletal muscle of rodents with subsequent morphological evaluation of muscle fibers.

\section{Material and Methods}

\section{Animal care and experimental groups}

The study was conducted in accordance with the Guide for Care and Use of Laboratory Animals (12). Twenty-six male Wistar rats weighing $269 \pm 26 \mathrm{~g}$ were used. The animals were housed in plastic cages in a room with controlled environmental conditions and had free access to water and standard food.

The animals were randomly divided into 3 groups: in group $I(N=6)$, the left hind limb was immobilized in order to maintain the soleus muscle in a fully shortened position for 3 weeks; in group II $(\mathrm{N}=6)$, the soleus was immobilized in the shortened position, but once a week the immobiliza- 
tion was removed and the muscle was maintained in a fully stretched position for 40 $\mathrm{min}$. After the stretching procedure, the hind limb was immobilized again in order to maintain the soleus in the shortened position; in group III the left soleus was submitted only to stretching (40 min) once a week $(\mathrm{N}=14)$. The right contralateral soleus of all animals was maintained intact and evaluated for comparison.

Rats were anesthetized with an intraperitoneal injection of xylazine $(12 \mathrm{mg} / \mathrm{kg})$ and ketamine $(95 \mathrm{mg} / \mathrm{kg})$ for the immobilization of soleus muscles during the stretching procedure and for muscle dissection and were then sacrificed with an overdose of anesthetic.

\section{Immobilization procedure}

To maintain the soleus muscle in the fully shortened position we used an alternative model developed in our laboratory for the immobilization of the rat hind limb, in which the left ankle joint was fixed in full plantar flexion (for details, see Ref. 13). The immobilization was adjusted daily to guarantee a full plantar position. The immobilization device is made of steel mesh and cotton materials, and has some advantages when compared to a plaster cast: it is cheaper and lighter and the same unit can be easily adjusted and used several times for the same animal or for animals of similar body weights. No skin ulceration or foot swelling was observed in the animals during the immobilization period. To stretch the soleus muscles, the left ankle was held in full dorsal flexion for 40 min by means of a piece of tape, as previously described by Williams (6).

\section{Histology and sarcomere measurements}

Three weeks after the beginning of the experiments all animals were anesthetized and weighed and both the right and left soleus muscles were carefully dissected free from surrounding tissue. Next, the tendons of the soleus were clamped with the muscle in the resting position, which was considered to be $\mathrm{L}_{0}$, as previously described by Ansved (4). Each soleus muscle was then divided longitudinally into two similar parts: the medial one was used for histology, while the lateral portion was used for the sarcomere measurements.

The method used in this study to determine the number and length of the sarcomeres along a single muscle fiber was developed by Williams and Goldspink (14). The muscle was fixed in the resting position ( $\mathrm{L}_{\mathrm{o}}$ length) for $3 \mathrm{~h}$ in $2.5 \%$ glutaraldehyde and then removed, placed in $30 \% \mathrm{HNO}_{3}$ for two days, and subsequently stored in $50 \%$ glycerol.

Five individual fibers of each whole soleus muscle were then teased out from tendon to tendon and mounted and their length was measured using a caliper rule. The number and length of the sarcomeres along a $300-\mu \mathrm{m}$ distance were quantified at different points of the middle region of each single fiber using a projection microscope (Axiolab, Carl Zeiss, Oberkochen, Germany). The total number of sarcomeres in each single muscle fiber was determined by the correlation between the number of sarcomeres identified along a distance of $300 \mu \mathrm{m}$ and the total fiber length (15). Since there are conflicting reports in the literature about the sarcomere length along the muscle fibers, particularly at the ends of the stretched fibers, in the present study it was assumed that the sarcomere length along the entire length of the muscle fiber was homogeneous (16-18).

For histology, the muscle was immediately frozen in isopentane pre-cooled in liquid nitrogen and stored in a freezer $\left(-80^{\circ} \mathrm{C}\right.$, Forma Scientific, USA). Serial cross-sections $(10 \mu \mathrm{m})$ were then obtained from the middle belly of the frozen muscles using a cryostat microtome (Microm HE 505, Germany) and stained with $1 \%$ toluidine blue/ $1 \%$ borax for the morphological evaluation of muscle fibers, as previously described (19-22). 


\section{Muscle fiber area}

The cross-sectional area of 100 muscle fibers randomly chosen in the central region of one cross-section of each soleus muscle was measured using a light microscope (Axiolab, Carl Zeiss) and software for morphology (Axiovision 3.0.6 SP4, Carl Zeiss).

\section{Statistical analysis}

The paired Student $t$-test was used to compare the data between the right and left soleus of the same animals in each group. Possible differences between groups were determined by ANOVA and the Duncan test, with the level of significance set at $\leq 0.05$.

\section{Results}

\section{Body and muscle weights}

After 21 days, both groups of immobilized animals showed a significant decrease in body weight when compared to the initial weight. Animals maintained immobilized for

Figure 1. Effect of one stretch a week on the rat soleus muscle. $A$, Muscle weight; $B$, serial sarcomere number; $C$, sarcomere length, and $D$, muscle fiber cross-sectional area. Data are reported as means $\pm \mathrm{SD}$. IM = the left soleus was immobilized in shortened position for 3 weeks $(\mathrm{N}=6) ; \mathrm{IM}+$ Stretch = the left soleus was immobilized for 3 weeks and stretched once a week $(N=6)$; Stretch $=$ the left soleus was stretched once a week for 3 weeks $(N=14)$. ${ }^{*} \mathrm{P}<$ 0.05 for right soleus compared to the contralateral one (Student $t$-test); $+\mathrm{P}<0.05$ for IM compared to the $I M+$ Stretch (ANOVA).

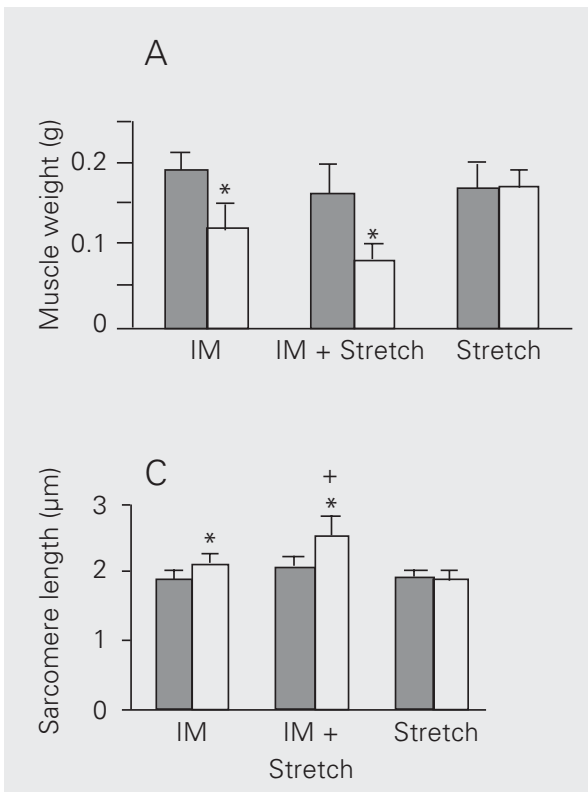

Right soleus
3 weeks lost $12 \pm 6 \%$ of their body weight (from $291 \pm 33$ to $256 \pm 29 \mathrm{~g}, \mathrm{P}=0.009$ ) and the group immobilized and stretched once a week also lost $12 \pm 5 \%$ of their weight (from $263 \pm 15$ to $231 \pm 18 \mathrm{~g}, \mathrm{P}=0.0005)$. The animals that were not immobilized, but were submitted to stretching once a week presented an increase of $16 \pm 1.9 \%$ in body weight compared to the initial weight (from $289 \pm 26$ to $343 \pm 26 \mathrm{~g}, \mathrm{P}<0.05$ ).

Soleus muscles immobilized for 3 weeks lost $44 \pm 6 \%$ of their weight when compared to the contralateral muscle $(0.13 \pm 0.04 v \mathrm{~s}$ $0.19 \pm 0.03 \mathrm{~g}$, respectively, $\mathrm{P}=0.002$; Figure 1A). Soleus muscles immobilized and stretched weekly also lost $43 \pm 5 \%$ of their weight $(0.08 \pm 0.02$ vs $0.16 \pm 0.04 \mathrm{~g}$, respectively, $\mathrm{P}<0.05)$. However, the non-immobilized group stretched once a week did not show a difference in muscle weight between the right and left soleus $(0.17 \pm 0.02$ vs 0.17 $\pm 0.03, \mathrm{P}=0.63$; Figure $1 \mathrm{~A})$.

\section{Histology}

Soleus muscles immobilized in the short-
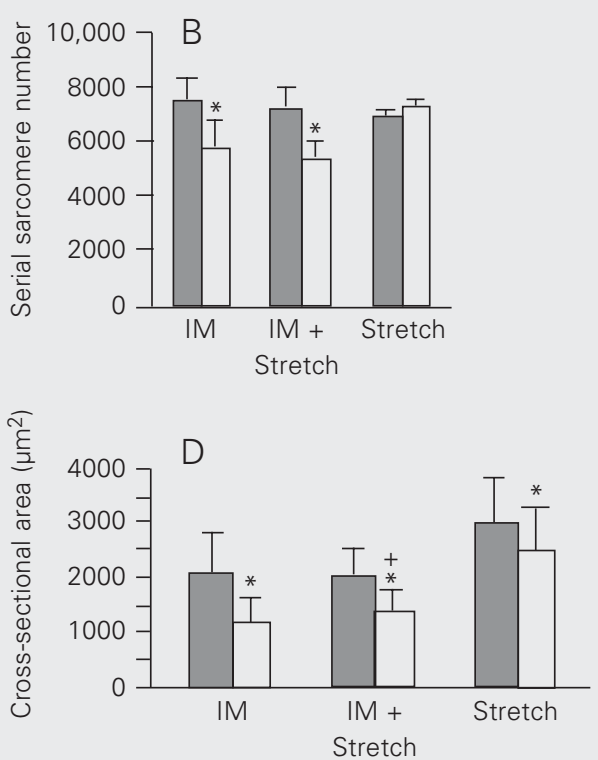

$\square$ Left soleus 
ened position for 3 weeks presented significant muscle fiber atrophy (Figure 2A) when compared to the contralateral soleus (Figure $2 \mathrm{~B})$. Note that the contralateral soleus showed fibers of normal aspect, but also with the abundant presence of regenerated fibers, such as split fibers and fibers with centralized nuclei. Similar muscle atrophy was observed in the left soleus submitted to immobilization and stretching once a week (Figure 2C), while the contralateral soleus presented normal and regenerated fibers (Figure 2D). Soleus muscle submitted only to stretching once a week (Figure 2E) and its contralateral muscle (Figure 2F) presented fibers of normal aspect. Figure 2E shows that stretched muscle also presented a small cross-sectional area compared to the contralateral one (Figure $2 \mathrm{~F})$.

\section{Sarcomere measurements}

The group submitted to 3 weeks of immobilization showed a decrease of $23 \pm 15 \%$ in the serial sarcomere number of the soleus muscle fibers compared to the contralateral muscles $(5748 \pm 1103$ vs $7517 \pm 797$, respectively, $\mathrm{P}=0.01$; Figure 1B). A decrease of 24 $\pm 14 \%$ in the sarcomere number of soleus muscle fibers was also observed in the group immobilized and stretched when compared to the contralateral one $(5376 \pm 613$ vs 7236 \pm 762 , respectively, $\mathrm{P}<0.05$ ). No significant difference in sarcomere number was detected between non-immobilized muscles stretched once a week and the contralateral muscles (7321 \pm 257 vs $6972 \pm 112, \mathrm{P}=0.06$; Figure 1B).

Soleus muscles immobilized in the shortened position also showed a change in sarcomere length, which was increased in the immobilized soleus compared to the contralateral muscle $(2.1 \pm 0.1 v s 1.9 \pm 0.1 \mu \mathrm{m}$, respectively, $\mathrm{P}=0.005$; Figure $1 \mathrm{C}$ ). The immobilized and stretched muscles also showed an increase in sarcomere length compared to the contralateral ones $(2.5 \pm 0.3 v s$
$2.1 \pm 0.1 \mu \mathrm{m}$, respectively, $\mathrm{P}<0.05$; Figure 1C). However, sarcomere length was larger in the immobilized and stretched muscles than in muscles submitted only to immobilization $(\mathrm{P}=0.0001$; Figure $1 \mathrm{C})$. In contrast, no change in sarcomere length was found in the fibers of soleus muscles submitted only to stretching compared to the contralateral

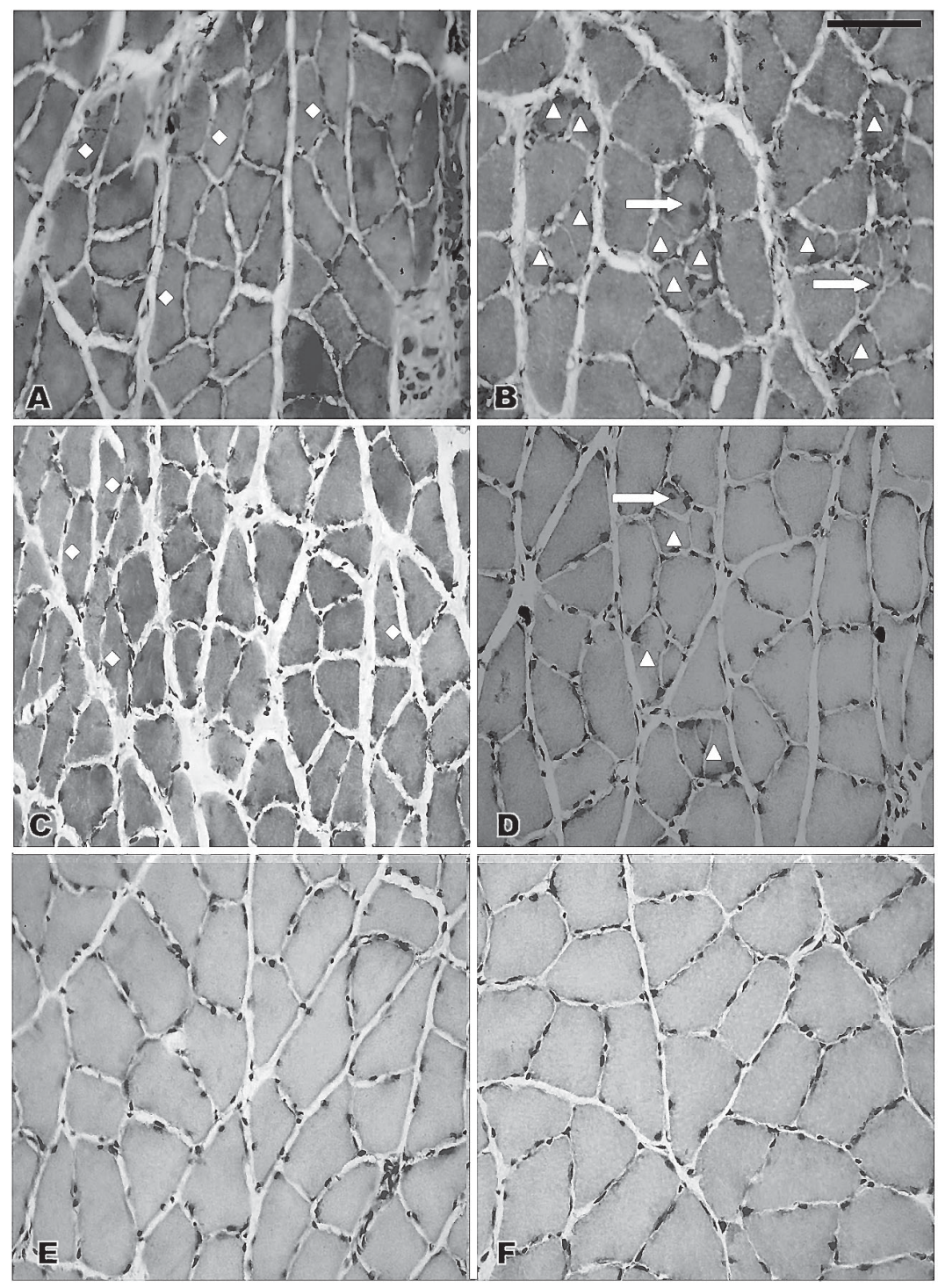

Figure 2. Effect of one stretch a week on muscle fiber morphology of rat soleus muscle. Cross-sections stained by toluidine blue. A, Atrophic muscle fibers (lozenges) of soleus muscle immobilized in the shortened position for 3 weeks, and $B$, contralateral muscle showing normal fibers, splits (triangles) and fibers with centralized nuclei (arrows). $C$, Soleus muscle immobilized for 3 weeks and stretched once a week also presents atrophy (lozenges) and the contralateral muscle (D) shows splits (triangles) and centralized nuclei in the muscle fibers (arrow). E, Soleus muscle stretched once a week, and $F$, contralateral muscle showing a normal aspect. Bar: $40 \mu \mathrm{m}$ for all panels. 
ones $(1.9 \pm 0.1$ vs $1.9 \pm 0.1 \mu \mathrm{m}$, respectively, $\mathrm{P}=0.6$; Figure 1C).

\section{Muscle fiber area}

Soleus muscles immobilized in the shortened position for 3 weeks showed a decrease in fiber cross-sectional area compared to the contralateral fibers $(1179 \pm 416$ vs $2084 \pm$ $728 \mu \mathrm{m}^{2}$, respectively, $\mathrm{P}<0.05$; Figure 1D). Muscle fiber atrophy was also observed in the soleus immobilized and stretched once a week compared to the contralateral one (1339 \pm 410 vs $1994 \pm 562 \mu \mathrm{m}^{2}$, respectively, $\mathrm{P}<$ 0.05; Figure 1D). Indeed, the immobilized and stretched muscle showed less atrophy compared to the muscle that was only immobilized ( $\mathrm{P}=0.0002$; Figure 1D).

On the other hand, it was surprising to find a significant decline in the cross-sectional area of muscle fibers between the left and right soleus of the animals submitted only to a weekly stretching session (2548 \pm 659 vs $2961 \pm 806 \mu \mathrm{m}^{2}$, respectively, $\mathrm{P}<$ 0.05 ; Figure 1D). Since this was an unexpected result, the same stretching protocol was tested again in a new group of rats $(\mathrm{N}=$ $8)$. The results of this second group confirmed the significant decrease in muscle fiber cross-sectional area in the soleus submitted only to one stretching session a week when compared to its contralateral muscle $\left(2518 \pm 692\right.$ vs $2700 \pm 600 \mu \mathrm{m}^{2}$, respectively, $\mathrm{P}<0.05$ ).

\section{Discussion}

The present study showed that passive stretching applied once a week for $40 \mathrm{~min}$ over a period of 3 weeks to the soleus of rats immobilized in the shortened position was not enough to prevent the loss of serial sarcomeres, but promoted significant protection against the muscle fiber atrophy. This result suggests that muscle stretching regulates the serial sarcomere number and the cross-sectional area of the muscle fibers by a different mechanism.

An unexpected and very interesting result of the present study was the significant reduction of muscle fiber area in the nonimmobilized soleus submitted to only one stretching session a week. This result was intriguing because it is recognized that muscle stretching is a very powerful stimulant of muscle growth and protein synthesis $(8,9)$, whereas the present results showed a crosssectional sarcopenic effect of stretching applied once a week. To our knowledge, this is the first report of a decrease in the crosssectional area of muscle fibers after one passive stretching session per week applied for 3 weeks to normal rat muscles. Thus, stretching also regulates the serial sarcomere number and the cross-sectional area of the muscle fibers in normal non-immobilized muscles differently.

Previous studies have demonstrated that the pathway for the addition of serial sarcomeres is different from that regulating the parallel sarcomere number. The nitric oxide (NO) derived from the neuronal isoform of NO synthase (nNOS) is a positive modulator of serial sarcomere addition (23), while the $\mathrm{Ca}^{+2} /$ calmodulin-dependent protein phosphatase calcineurin and insulin-like growth factor 1 (IGF-1), by activating the phosphatidyl-inositol 3-kinase (PI3k)-Akt (a serinethreonine kinase) pathways, appear to regulate skeletal muscle hypertrophy $(24,25)$. On the other hand, the ubiquitin-proteasome pathway regulates the binding and degradation of ubiquitinated proteins in the process of atrophy (26).

The absence of difference in absolute muscle weight between the right and left soleus in the group submitted only to stretching could be the consequence of the small amount of muscle fiber atrophy found, which probably was not enough to produce significant changes in absolute muscle weight. It is well known that muscle fiber area is a better measurement than absolute muscle weight for the evaluation of skeletal muscle tro- 
phism.

An interesting study by Williams (2) showed that when one stretching session was applied daily for $30 \mathrm{~min}$ to muscles immobilized in the shortened position, it prevented the loss of both muscle weight and serial sarcomere number. A comparison of our results with those reported by Williams (2) indicates that the frequency and duration of stretching applied to muscle immobilized in the shortened position is important to preserve muscle mass and the muscle fiber length, i.e., a daily session of $30 \mathrm{~min}$ of stretching, but not one 40-min stretching session a week, preserves muscle weight and serial sarcomere number.

The increase in sarcomere length in the shortened muscles has been associated with the reduction of serial sarcomere number, which involves an adjustment in sarcomere length for the muscle to develop its maximal tension (1). Thus, in muscles immobilized in the shortened position, sarcomeres are lost and the remaining sarcomeres are stretched to a length that enables the muscle to develop its maximum tension in the immobilized position (1).

The present study showed a significant increase in sarcomere length in the soleus muscle fibers of both groups of immobilized animals when compared to the contralateral muscles. Also, the sarcomere length was higher in the immobilized and stretched muscles compared to the muscles only immobilized. However, no difference was found in the loss of serial sarcomere number between the immobilized groups.

The significant reduction of body weight found in the immobilized animals was prob- ably due to the disuse and muscle fiber atrophy of the immobilized hind limb. However, the loss of body weight occurring with the use of our immobilization device was less pronounced than that observed by others with the use of a plaster cast for muscle immobilization (4).

It is well known that muscle overuse, especially in sedentary animals, can induce muscle fiber injury (27). Some classical signs of the lesion/regeneration cycle are the presence of split fibers and centralized nuclei. Previous studies have shown that split fibers are provoked in response to stress in hypertrophied fibers (28). Thus, the presence of regenerated fibers identified in the contralateral soleus muscle is probably associated with the injury caused by its overuse.

One session of passive stretching applied once a week for 40 min to the soleus muscle of rats immobilized in the shortened position was not enough to prevent the loss of muscle weight and serial sarcomere number, but provided significant protection against muscle fiber atrophy. In contrast, a significant reduction in muscle fiber area was found in the non-immobilized soleus submitted to only one stretching session a week. These results suggest that muscle stretching regulates the serial sarcomere number and the cross-sectional area of muscle fibers by different mechanisms.

\section{Acknowledgments}

The authors are grateful to Dr. Jorge Oishi for help with the statistical analysis and to Tereza F. Piassi for technical assistance during the experiments.

\section{References}

1. Williams PE \& Goldspink G (1978). Changes in sarcomere length and physiological properties in immobilized muscle. Journal of Anatomy, 127: 459-468.

2. Williams PE (1990). Use of intermittent stretch in the prevention of serial sarcomere loss in immobilized muscle. Annals of the Rheu- matic Diseases, 49: 316-317.

3. Williams PE \& Goldspink G (1984). Connective tissue changes in immobilized muscle. Journal of Anatomy, 138: 343-350.

4. Ansved T (1995). Effects of immobilization on the rat soleus muscle in relation to age. Acta Physiologica Scandinavica, 154: 291-302. 
5. Kannus P, Jozsa L, Järvinen TLN, Kvist M, Vieno T, Järvinen TAH, Natri A \& Järvinen M (1998). Free mobilization and low- to highintensity exercise in immobilization-induced muscle atrophy. Journal of Applied Physiology, 84: 1418-1424.

6. Williams PE (1988). Effect of intermittent stretch on immobilized muscle. Annals of the Rheumatic Diseases, 47: 1014-1016.

7. Gajdosik RL (2001). Passive extensibility of skeletal muscle: review of literature with clinical implications. Clinical Biomechanics, 16: 87101.

8. Goldspink G (1999). Molecular mechanism involved in the determination of muscle fiber mass and phenotype. Advances in Exercise and Sports Physiology, 5: 27-39.

9. Goldspink G, Williams P \& Simpson H (2002). Gene expression in response to muscle stretch. Clinical Orthopaedics and Related Research, 403: 146-152.

10. Wallin D, Ekiblom B, Grahn R \& Nordenborg T (1985). Improvement of muscle flexibility: a comparison between two techniques. American Journal of Sports Medicine, 13: 263-268.

11. Frontera WR, Dawson DM \& Slovik DM (1999). Exercise in Rehabilitation Medicine. Human Kinetics Publishers, Inc., Champaign, IL, USA, 99

12. National Research Council (1996). Guide for the Care and Use of Laboratory Animals. National Academy Press, Washington, DC, USA.

13. Coutinho EL, Gomes ARS, França CN \& Salvini TF (2002). A new model for the immobilization of the rat hind limb. Brazilian Journal of Medical and Biological Research, 35: 1329-1332.

14. Williams PE \& Goldspink G (1971). Longitudinal growth of striated muscle fibers. Journal of Cell Science, 9: 751-761.

15. Williams PE, Catanese T, Lucey EG \& Goldspink G (1988). The importance of stretch and contractile activity in the prevention of connective tissue accumulation in muscle. Journal of Anatomy, 158: 109-114.

16. Tardieu C, Tabary JC, Tabary C \& Huet de La Tour E (1977). Comparison of the sarcomere number adaptation in young and adult animals. Journal de Physiologie, 73: 1045-1055.

17. Matano T, Tamai K \& Kurokawa T (1994). Adaptation of skeletal muscle in limb lengthening: a light diffraction study on the sarcomere length in situ. Journal of Orthopaedic Research, 12: 193-196.

18. Tamai K, Kurokawa T \& Matsubara I (1989). In situ observation of adjustment of sarcomere length in skeletal muscle under sustained stretch. Nippon Seikeigeka Gakkai Zasshi, 63: 1558-1563.

19. Salvini TF, Morini CC, Selistre de Araujo HS \& Ownby CL (1999). Long-term regeneration of fast and slow murine skeletal muscles after induced injury by ACL myotoxin isolated from Agkistrodon contortrix laticinctus (broad-banded copperhead) venom. Anatomical Record, 254: 521-533.

20. Oliveira NML, Parizzotto NA \& Salvini TF (1999). GaAs (904-Nm) laser radiation does not affect muscle regeneration in mouse skeletal muscle. Lasers in Surgery and Medicine, 25: 13-21.

21. Minamoto VB, Grazziano CR \& Salvini TF (1999). Effect of single and periodic contusion on the rat soleus muscle at different stages of regeneration. Anatomical Record, 254: 281-287.

22. Minamoto VB, Bunho SR \& Salvini TF (2001). Regenerated skeletal muscle of rat after periodic contusions. Brazilian Journal of Medical and Biological Research, 34: 1447-1452.

23. Koh TJ \& Tidball JG (1999). Nitric oxide synthase inhibitors reduce sarcomere addition in rat skeletal muscle. Journal of Physiology, 519: 189-196.

24. Dunn SE, Burns JL \& Michel RN (1999). Calcineurin is required for skeletal muscle hypertrophy. Journal of Biological Chemistry, 274: 21908-21912.

25. Glass DJ (2003). Molecular mechanisms modulating muscle mass. Trends in Molecular Medicine, 9: 344-350.

26. Kandarian SC \& Stevenson EJ (2002). Molecular events in skeletal muscle during disuse atrophy. Exercise and Sport Sciences Reviews, 30: 111-116

27. Wernig A, Salvini TF \& Irintchev A (1991). Axonal sprouting and changes in fibre types after running-induced muscle damage. Journal of Neurocytology, 20: 903-913.

28. Carpenter S \& Karpati G (1984). Pathology of Skeletal Muscle. Churchill Livingstone, New York, Edinburgh, London, Melbourne, 125-126. 